\title{
Effect upon hospital activity of the application of a continued care model centered on patients with inflammatory bowel disease
}

\author{
Francesc Casellas-Jordá, Natalia Borruel-Sainz, Antonio Torrejón-Herrera and Isabel Castells \\ Unitat d'Atenció Crohn-Colitis (UACC). Hospital Universitari Vall d'Hebron. CIBERehd. Barcelona, Spain
}

\begin{abstract}
Background: since 1999, the Crohn-Colitis Care Unit (UACC) has been dedicated to the integral management of patients with Crohn's disease (CD) and ulcerative colitis (UC). The working methodology of the UACC is based on personalized, continued, nonphysical presence, open access and patient-centered care. From its creation, the UACC has experienced an increase in the number of its users and outpatient services. However, the impact of the activity of the UACC upon patient hospitalization is not known.

Objectives: to determine the hospital activity related to $\mathrm{CD}$ and UC, and correlate it to the activity of the UACC.

Methods: a retrospective evaluation was made of the physical presence and non-presence activities of the UACC from January 1999 to December 2008, and of the hospital admissions and mean durations of stay due to $\mathrm{CD}$ and UC during that same time period.

Results: the number of attended patients and of presence and non-presence activities of the UACC has gradually increased. This increase contrasts with the number of annual hospital admissions, which has remained stable during the study period, with 200-300 admissions/year. Consequently, the hospitalized patients / UACC registered patients ratio has decreased from 0.36 at the start of the study period to 0.14 at the end. The median hospital stay has also decreased, from 11 days at the start of the study period to 8 days at the end.

Conclusions: the UACC allows effective management of IBD patient care, since it is able to attend the needs of more patients without increasing the number of admissions, and shortening the duration of hospital stay.
\end{abstract}

This work was supported in part by grants from the Generalitat de Catalunya (RE: 2001SGR00389) and the Centro de Investigación Biomédica en Red de Enfermedades Hepáticas y Digestivas (CIBERehd, Spain). CIBERehd is funded by the Instituto de Salud Carlos III, Spain.

Received: 08-06-11.

Accepted: 01-09-11.

Correspondence: Francesc Casellas. UACC. Hospital Universitari Vall d'Hebron. Pso. Vall d'Hebron, 119. 08035 Barcelona, Spain.

e-mail: fcasellas@vhebron.net
Key Words: Inflammatory bowel disease. Continued care. IBD unit. Ulcerative colitis. Crohn's disease. Hospital admissions.

Casellas-Jordá F, Borruel-Sainz N, Torrejón-Herrera A, Castells I. Effect upon hospital activity of the application of a continued care model centered on patients with inflammatory bowel disease. Rev Esp Enferm Dig 2012; 104: 16-20.

\section{INTRODUCTION}

Inflammatory bowel disease (IBD) comprises two chronic inflammatory illnesses: Crohn's disease (CD) and ulcerative colitis (UC). IBD has important medical and social repercussions for several reasons. On one hand, these disorders are increasingly common, and in our setting their annual incidence per 100,000 inhabitants has increased from 1.9 to 8.0 in the case of $\mathrm{CD}$, and from 3.8 to 9.5 in the case of UC (1-3). On the other hand, IBD particularly affects fully productive and reproductive young adults. Such individuals suffer a chronic disease with both digestive and non-digestive symptoms, and recurrent flare-ups which in turn can be accompanied by a range of complications. In addition, patients with IBD must receive continued and complex treatment, with potential serious adverse effects. On the other hand, despite such treatment, resective surgery may prove necessary, with long-term consequences such as those associated with the need for ostomies. All these factors explain why the management of IBD patients is complex, requiring a multidisciplinary approach to management on a continued basis and centered on the needs of each individual patient.

The introduction of specialized Units is able to improve the healthcare of patients with IBD, as reflected by improved quality of life, adherence to therapy, patient satisfaction, fewer hospital admissions and a lesser use of corticosteroids (4). 
In 1999 the Crohn-Colitis Care Unit (UACC) (Hospital Universitari Vall d'Hebron, Barcelona, Spain) was created as a service for promoting the health of patients with IBD. The UACC is composed of a team of healthcare professionals -physicians and others- with expertise in the field of IBD, who offer integral attention to patient demands. The working methodology of the UACC is based on personalized, continued, multidisciplinary, non-physical presence, open access and patient-centered care. To this effect, the UACC has its own specific facilities, an open telephone line attended by professionals with training in IBD, a specific computer-based registry of activities, and a website (http://www.ua-cc.org) with a range of interactive services offering users a guided and tutored way to satisfy their information needs. Thus, the UACC model is not a monographic consulting center but rather a resources coordinating unit that attends demands (such as by telephone), programs visits for participation in health education projects, or arranges appointments for medical visits upon demand (5). The introduction of this service aimed to address the deficiencies detected by the patients themselves, such as insufficient information, a lack of attention to the patient environment, and deficient accessibility and fluidness in the use of healthcare resources (6).

An analysis of the activity of the UACC in its first years of functioning has revealed a progressive increase in both the physical presence and non-presence activity of the Unit, and in website activity -this running parallel to an increase in the number of patients documented in the computer registry (7). In response to the application of visual analog scales specifically designed to assess user satisfaction with the UACC, the patients expressed a high degree of satisfaction (score 9/10) in reference to areas such as healthcare continuity, convenience, accessibility, courtesy and professional competence (8). The availability of a specialized continued and IBD patient-centered healthcare tool such as the UACC model improves patient care and satisfaction, though it remains to be established whether it is also accompanied by an excessive burden in terms of hospital resource utilization. The present study was therefore designed to examine the evolution of hospital resource utilization, the number of hospital admissions and the duration of hospital stay since the UACC came into operation.

\section{METHODS}

A retrospective, comparative observational study was made of the activity of the UACC and of the number of hospital admissions and the duration of hospital stay generated by patients with $\mathrm{CD}$ and UC.

According to data from 2008, the General Area of the Hospital Universitari Vall d'Hebron has 743 hospitalization beds corresponding to the clinical and surgical specialties, excluding the areas of Traumatology-Rehabilitation and MaternalPediatric Care. This number of beds remained stable during the period of the study. The Hospital attends a population of 453,196 inhabitants in Barcelona (51\% of all hospital discharges correspond to the reference area), the rest of Catalonia

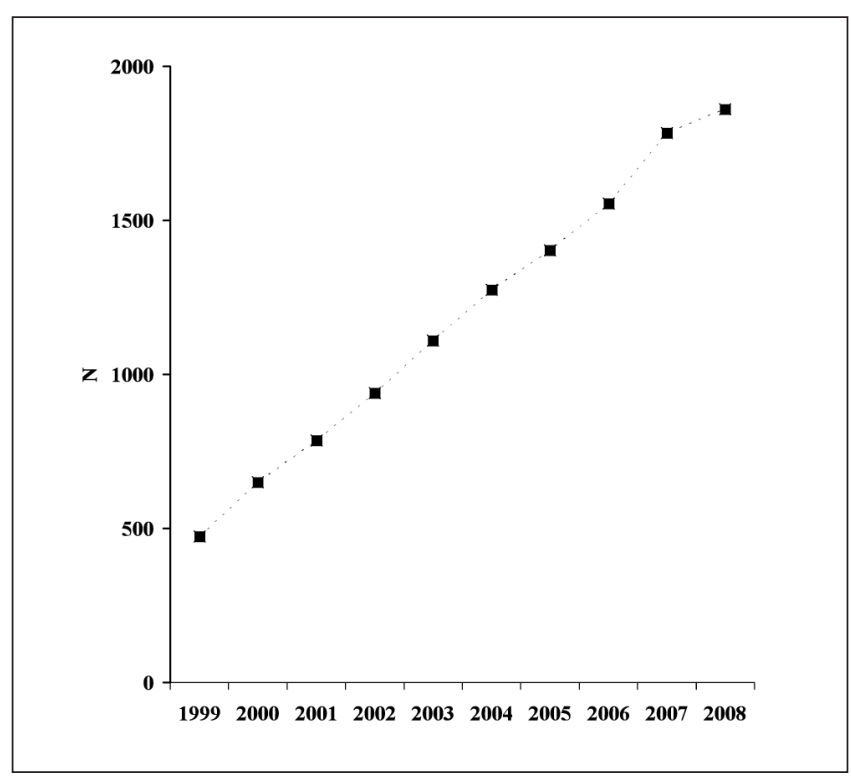

Fig. 1. Cumulative number of registered patients in the UACC during the analyzed period.

(45\% of all hospital discharges), and the rest of Spain (2\%).

\section{Procedure}

A review was made of the UACC activity registry data and clinical and surgical admissions records of the Hospital Universitari Vall d'Hebron for the period between 1 January 1999 and 31 December 2008, involving admitted patients with diagnosis-related group classification codes corresponding to $\mathrm{CD}$ or UC (both principal and secondary diagnoses, and independently of disease location) independently of the Department. In calculating the number of activities in the UACC, we did not include outpatient clinic visits or hospital interconsultations.

\section{Statistical analysis}

According to the Kolmogorov-Smirnov test, some values did not conform to normality. Thus, variables were described as absolute numbers or medians. Dunn's test was used for all pair wise comparisons following rank-based ANOVA where necessary.

\section{RESULTS}

\section{Users registered in the UACC}

The number of users registered in the UACC has progressively increased each year, reaching 1,784 at the end of the study period (Fig. 1). The Unit has registered 849 


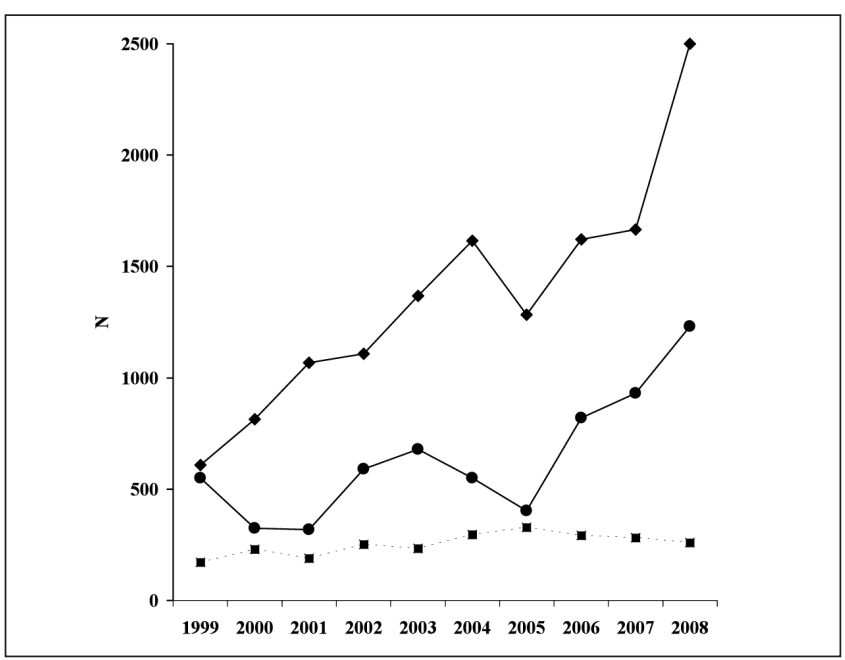

Fig. 2. The annual number of physical presence (black circles) and nonpresence activities (black squares) has progressively increased each year, while the number of hospital admissions due to IBD has remained stable (squares on broken line).

patients with $\mathrm{CD}$ and 893 with UC (the rest corresponding to indeterminate disease), of which 880 were males and 904 females. Most of the subjects were young and only 380 $(21 \%)$ were over 50 years of age.

\section{Healthcare activity in the UACC and hospital admissions}

The physical presence and non-presence activities of the UACC have increased each year during the first 10 years of the UACC. The increase in activity has been particularly notorious in the last four years, as can be seen in figure 2 . In contrast,

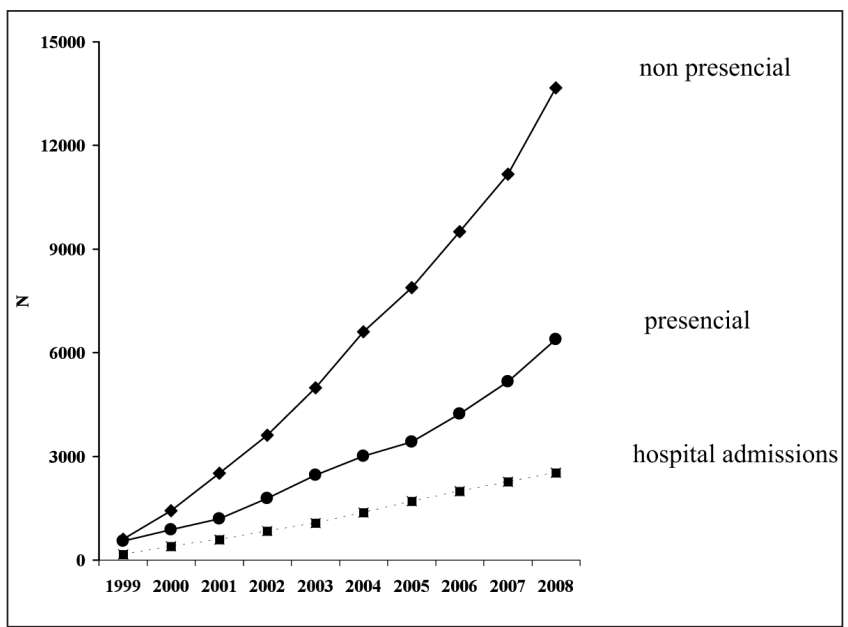

Fig. 3. The cumulative annual number of physical presence (black circles) and non-presence activities (black squares) has increased more markedly than the number of hospital admissions due to IBD (squares on broken line).

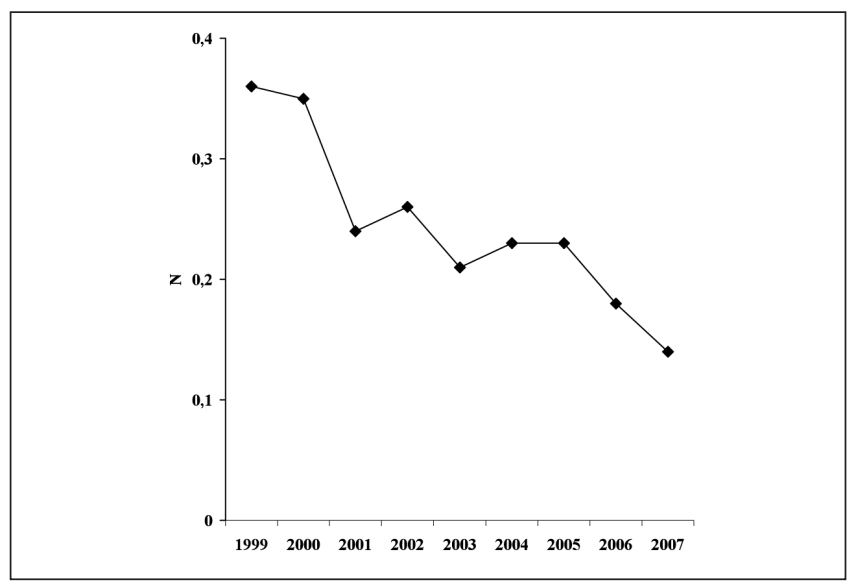

Fig. 4. The annual ratio between the number of hospitalized patients and the number of UACC registered patients has decreased progressively over the first 10 years of activity of the Unit.

the annual number of hospital admissions has remained stable, with between 170 and 329 admissions a year. As a consequence of the increase in activity of the UACC, the slope of the cumulative interventions curve is greater for UACC activity than for the number of patients admitted to hospital. In addition, the slope of the curve is greater for non-physical presence activity than for physical presence activity of the UACC (Fig. 3).

The progressive increase in the activity of the UACC has not been accompanied by an increase in hospital activity. This is confirmed by the progressive and marked decrease in the ratio between the annual hospital admissions and the patients registered in the UACC (Fig. 4).

Another aspect that has been examined in relation to the effect of the UACC upon hospital activity is the duration of hospital stay. Figure 5 shows that the duration of hospital stay (in days) decreased from a median of 11 days at the

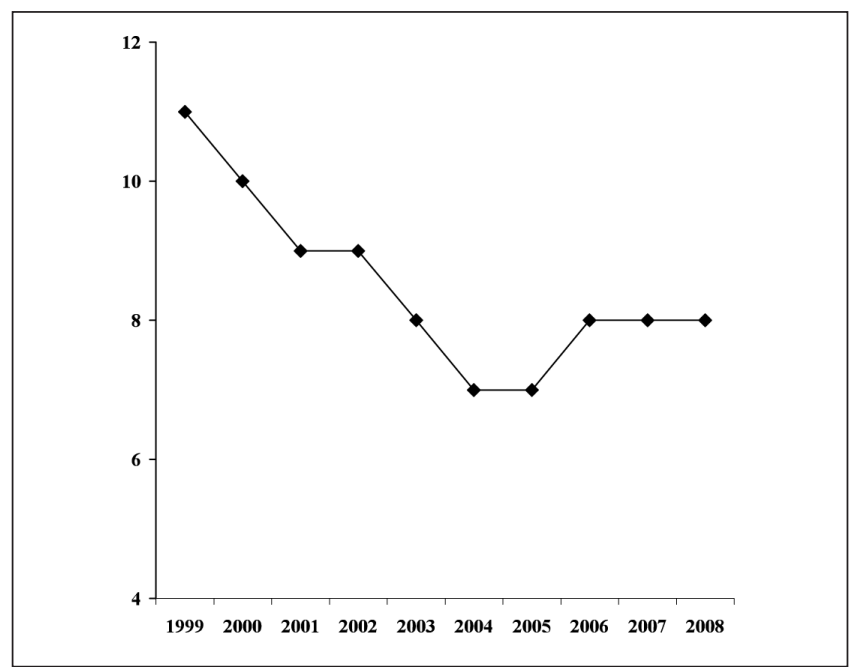

Fig. 5. The median duration of hospital stay has decreased since implantation of the UACC model in the Hospital. 
start of the study period to 8 days at the end of follow-up. The change between the first year of operation of the UACC and the end of the study period proved statistically significant (Dunn's test, $\mathrm{p}<0.05$ ).

\section{DISCUSSION}

Inflammatory bowel disease (IBD) is increasingly common in industrialized western countries (9). This fact, and the clinical repercussions and therapeutic complexity of the disease, generate important personal and social costs. One of the main factors contributing to the cost and healthcare resources consumption associated with IBD refers to hospital admissions (10). An added consideration is the fact that despite the improvements in the medical treatment of IBD, the hospital admission rate per inhabitant attributable to the disease continues to increase (11-14). For this reason we considered it to be very interesting to evaluate the influence upon hospital admission of the UACC model based on patient-centered, continued, multidisciplinary, non-physical presence, open access care involving specialized telephone-based attention.

In order to improve the care of patients with IBD, and in concordance with the current biopsychosocial approach to the management of chronic diseases, Units or Departments have been designed to offer care specifically centered on the patient. The incorporation of training programs with personalized patient self-management plans and telephone follow-up and appointments upon demand does not significantly modify the course of the disease or patient quality of life, but the affected patients nevertheless find this approach more convenient and prefer it to the conventional system involving programmed follow-up (15). Over a period of one year, follow-up centered on the patient with open telephone access has shown that the patients feel more sure of themselves in being able to cope with the disease, and visit the hospital less often (16). Studies have been made to determine whether such patient-centered care increases the associated costs, based on cost-effectiveness analyses and estimations of the quality-adjusted life years gained (QALYs). One such study (17) has shown that during the first year of operation of the self-management program, a saving of $148 £$ is obtained per patient, with a probability of around $63 \%$ that the whole system approach to self-management is cost-effective, assuming a willingness to pay up to 30,000 £ for an additional QALY. However, little information is available on the results of this type of healthcare model over a longer term.

A retrospective analysis has been made of the progression of the number of admissions from the start of activity of the UACC, contemplating all admissions -both clinical and surgical. The results of the present study show that from the creation of the UACC the number of patients attended has increased, but hospital admissions has remained stable, with no progressive increments such as those reported by studies conducted in the United States and Canada. This finding contrasts with the observed increase in the number of patients registered and the healthcare activity -both physical presence care in the UACC and patient attention by telephone. As a result, the ratio between the number of hospital admissions and the patients registered in the UACC has decreased markedly and progressively. The present study does not allow us to conclude that the absence of an increase in the number of admissions is a direct consequence of the activity of the UACC, since it has not been possible to establish the influence of other factors such as changes in the criteria for hospital admission or the effect of recently introduced drugs (18). However, we consider that more effective patient management in the Emergency Department from the UACC, and the fact that the latter guarantees close and specialized follow-up, allowing even the use of second-line drug treatments, make it unnecessary to decide hospital admissions which otherwise would have been unavoidable.

Similar results have been obtained in relation to the duration of hospital admission. In the first 7 years we observed a gradual shortening of hospital stay, followed by stabilization in the last three years. In this context, the shortening of hospital stay has been statistically significant. The influence of the UACC in relation to the shortening of hospital stay may be related to the fact that patient discharge can be decided earlier, since follow-up on an outpatient basis is more fluid. Due to the retrospective nature of the study, we here again cannot exclude the influence of other factors such as for example improved management of hospital resources, treatment innovations, etc. Thus, the effect of factors such as the update of diagnostic and therapeutic protocols, new biologic treatments, the reduction in the delay of explorations has not been evaluated. The functioning of the UACC coincides with the introduction of the biologic antiTNF agents being difficult to individualize the effect of the UACC from the effect of antiTNF treatment because this treatment is given and controlled in the UACC.

In conclusion, implantation in the Hospital of the UACC model allows effective management of IBD patient care, since it has been possible to attend the needs of more patients without increasing the number of admissions, and shortening the duration of hospital stay.

\section{REFERENCES}

1. Pajares JM, Gisbert JP. Epidemiology of inflammatory bowel disease in Spain. A systematic review. Rev Esp Enferm Dig 2001;93:9-20.

2. López-Serrano P, Pérez-Calle JL, Carrera-Alonso E, Pérez-Fernández T, Rodríguez-Caravaca G, Boixeda-de-Miguel D, et al. Epidemiologic study on the current incidence of inflammatory bowel disease in Madrid. Rev Esp Enferm Dig 2009;101:768-72.

3. Arin Letamendia A, Borda Celaya F, Burusco Paternain MJ, Prieto Martínez C, Martínez Echeverría A, Elizalde Apestegui I, et al. High incidence rates of inflammatory bowel disease in Navarra (Spain). Results of a prospective, population-based study. Gastroenterol Hepatol 2008;31:111-6.

4. Phan VA, van Langenberg DR, Grafton R, et al. Introduction of a dedicated IBD service quantitatively and qualitatively improves outcomes in less than 18 months: a prospective cohort study in a large metropolitan centre. Gastroenterology 2010;138(Supl. 1): S318.

5. Fontanet G, Casellas F, Malagelada JR. The Crohn-Colitis care unit: analysis of the first 3 years of activity. Gac Sanit 2004;18:483-5. 
6. Casellas F, Fontanet G, Borruel N, Malagelada JR. The opinion of patients with inflammatory bowel disease on healthcare received. Rev Esp Enferm Dig 2004;96:174-84.

7. Torrejón A, Masachs M, Borruel N, Castells I, Castillejo N, Malagelada JR, et al. Aplicación de un modelo de asistencia continuada en la enfermedad inflamatoria intestinal: la Unidad de Atención Crohn-Colitis. Gastroenterol Hepatol 2009;32:77-82.

8. Masachs M, Casellas F, Borruel N, Torrejón A. Castells I, Malagelada JR. Validation of the Spanish version of a questionnaire to measure quality of care through the eyes of patients with inflammatory bowel disease (QUOTE-IBD). Inflamm Bowel Dis 2010;16:982-92.

9. Weiss ST. The hygiene hypothesis and allergic diseases. New Engl J Med 2002;347:930-1

10. Casellas F, Panés J, García-Sánchez V, Ginard D, Gomollón F, Hinojosa $\mathrm{J}$, et al. A. Costes médicos directos de la enfermedad de Crohn en España. Pharmacoeconomics-Spanish Research Articles 2010;7:38-46.

11. Bewtra M, Su C, Lewis JD. Trends in hospitalization rates for inflammatory bowel disease in the United States. Clin Gastroenterol Hepatol 2007;5:597-601.

12. Nguyen GC, Sam J, Murthy SK, Kaplan GG, Tinmouth JM, LaVeist TA Hospitalizations for inflammatory bowel disease: profile of the uninsured in the United States. Inflamm Bowel Dis 2009;15:726-33.
13. Nguyen GC, Steinhart AH. Nationwide patterns of hospitalizations to centers with high volume of admissions for inflammatory bowel disease and their impact on mortality. Inflamm Bowel Dis 2008;14:1688-94.

14. Wang YR, Cangemi JR, Picco MF. Changing Trends of Hospitalizations for Inflammatory Bowel Disease in the United States, 1993-2007. Gastroenterology 2010;138(Supl. 1):S531-S532.

15. Robinson A, Thompson DG, Wilkin D, Roberts C, for the Northwest Gastrointestinal Research Group. Guided self-management and patientdirected follow-up of ulcerative colitis: a randomised trial. Lancet 2001;358:976-81.

16. Kennedy AP, Nelson E, Reeves D, Richardson G, Roberts C, Robinson $\mathrm{A}$, et al. A randomised controlled trial to assess the effectiveness and cost of a patient orientated self management approach to chronic inflammatory bowel disease. Gut 2004;53:1639-45.

17. Richardson G, Sculpher M, Kennedy A, Nelson E, Reevers D, Roberts $\mathrm{C}$, et al. Is self-care a cost-effective use of resources? Evidence from a randomized trial in inflammatory bowel disease. J Health Serv Res Policy 2006;11:225-30.

18. Taxonera C, Rodrigo L, Casellas F, Calvet X, Gómez-Camacho F, Ginard D, et al. Infliximab maintenance therapy is associated with decreases in direct resource use in patients with luminal or fistulizing Crohn's disease. J Clin Gastroenterol 2009;43:950-6. 